\title{
HIGH-VELOCITY FEATURES: A UBIQUITOUS PROPERTY OF TYPE Ia SUPERNOVAE
}

\author{
P. A. Mazzali, ${ }^{1,2}$ S. Benetti,${ }^{3}$ G. Altavilla, ${ }^{4}$ G. Blanc, ${ }^{3}$ E. Cappellaro, ${ }^{5}$ N. Elias-Rosa, ${ }^{3}$ G. Garavini, ${ }^{6,7}$ A. Goobar, ${ }^{7}$ \\ A. Harutyunyan, ${ }^{3}$ R. Kotak,${ }^{8}$ B. Leibundgut, ${ }^{9}$ P. Lundqvist,${ }^{10}$ S. Mattila,,${ }^{10}$ J. Mendez, ${ }^{4}$ S. Nobili, ${ }^{6,7}$ R. Pain, ${ }^{6}$ \\ A. Pastorello, ${ }^{2,3}$ F. Patat, ${ }^{9}$ G. Pignata, ${ }^{9}$ Ph. Podsiadlowski, ${ }^{11}$ P. Ruiz-Lapuente, ${ }^{4}$ M. Salvo, ${ }^{12}$ B. P. Schmidt ${ }^{12}$ \\ J. Sollerman, ${ }^{10}$ V. Stanishev, ${ }^{7}$ M. Stehle, ${ }^{2}$ C. Tout,${ }^{13}$ M. Turatto, ${ }^{3}$ and W. Hillebrandt ${ }^{2}$ \\ Received 2005 January 27; accepted 2005 February 24; published 2005 March 11
}

\begin{abstract}
Evidence of high-velocity features (HVFs) such as those seen in the near-maximum spectra of some Type Ia supernovae (SNe Ia; e.g., SN 2000cx) has been searched for in the available SN Ia spectra observed earlier than 1 week before $B$ maximum. Recent observational efforts have doubled the number of SNe Ia with very early spectra. Remarkably, all SNe Ia with early data (seven in our Research Training Network sample and 10 from other programs) show signs of such features, to a greater or lesser degree, in Ca II IR and some also in the Si II $\lambda 6355$ line. HVFs may be interpreted as abundance or density enhancements. Abundance enhancements would imply an outer region dominated by Si and Ca. Density enhancements may result from the sweeping up of circumstellar material (CSM) by the highest velocity SN ejecta. In this scenario, the high incidence of HVFs suggests that a thick disk and/or a high-density companion wind surrounds the exploding white dwarf, as may be the case in single degenerate systems. Large-scale angular fluctuations in the radial density and abundance distribution may also be responsible: this could originate in the explosion and would suggest a deflagration as the more likely explosion mechanism. CSM interaction and surface fluctuations may coexist, possibly leaving different signatures on the spectrum. In some SNe, the HVFs are narrowly confined in velocity, suggesting the ejection of blobs of burned material.
\end{abstract}

Subject heading: supernovae: general

\section{INTRODUCTION}

The importance of Type Ia supernovae (SNe Ia) for cosmological studies has been the motivation for increased efforts to understand how these bright explosions work and how their observational properties depend on the physical processes involved. Three-dimensional modeling of the thermonuclear explosion of a CO white dwarf reaching the Chandrasekhar limit holds the promise to describe successfully how SNe Ia are born. On the observational side, several groups are trying to follow nearby $\mathrm{SNe}$ Ia with an increased temporal coverage, so that explosion models can be tested and the range of observational SN properties fully described.

In particular, it is now agreed that it is important to observe $\mathrm{SNe}$ Ia as early as possible. Early observations make it easier to distinguish SNe Ia from SNe Ic and allow firmer constraints

${ }^{1}$ INAF-Osservatorio Astronomico, Via Tiepolo 11, 34131 Trieste, Italy.

${ }^{2}$ Max-Planck-Institut für Astrophysik, Karl-Schwarzschild-Strasse 1, 85748 Garching, Germany.

${ }^{3}$ INAF-Osservatorio Astronomico di Padova, Vicolo dell'Osservatorio 5, 35122 Padua, Italy.

${ }^{4}$ Department of Astronomy, University of Barcelona, Avenida Diagonal 647, 8028 Barcelona, Spain.

${ }^{5}$ INAF-Osservatorio Astronomico di Capodimonte, Via Moiarielle 16, 80131 Naples, Italy.

${ }^{6}$ Laboratoire de Physique Nucléaire et de Haute Energies, CNRS-IN2P3, Université Paris VI and VII, Paris, France.

${ }^{7}$ Department of Physics, Stockholm University, Saltsjoebaden, SE-10691 Stockholm, Sweden.

${ }^{8}$ Blackett Laboratory, Imperial College of Science, Technology and Medicine, Prince Consort Road, London SW7 2BZ, England, UK.

${ }^{9}$ European Southern Observatory, Karl-Schwarzschild-Strasse 2, 85748 Garching, Germany.

${ }^{10}$ Stockholm Observatory, SE-10691 Stockholm, Sweden.

${ }^{11}$ Department of Astronomy, Oxford University, Keble Road, Oxford OX1 3NP, England, UK.

${ }^{12}$ Mount Stromlo and Siding Spring Observatory, Private Bag, Weston Creek P.O., Canberra ACT 2601, Australia.

${ }^{13}$ Institute of Astronomy, University of Cambridge, Madingley Road, Cambridge CB3 OHA, England, UK. to be placed on the actual explosion date (e.g., Riess et al. 1999; Mazzali \& Schmidt 2000), which is important for the use of SNe Ia in cosmology. Moreover, early spectra explore the outer layers of the SN ejecta. These layers may hold traces of the properties of the progenitor system, whose nature may thereby be revealed (e.g., Lentz et al. 2000).

As the number of high signal-to-noise ratio observations increases, a few cases have been reported of SNe Ia with overall normal properties but displaying high-velocity absorption lines, in particular, of the Ca II IR triplet. The first such features identified were Ca II and Fe II lines in SN 1994D (Hatano et al. 1999). Further evidence came from SN 2000cx (Li et al. 2001), which showed two strong sets of Ca II IR lines, at different high velocities, in addition to the photospheric component. High-velocity features (HVFs) were also observed in SN 2001el (Wang et al. 2003), SN 2003du (Gerardy et al. 2004), and SN 1999ee. For SN 1999ee, Mazzali et al. (2005) note that high-velocity absorption is present not only in Ca II IR but also in the strong Si II $\lambda 6355$ line. HVFs may be indicative of interaction with a circumstellar medium (Gerardy et al. 2004), of an intrinsic three-dimensional structure of the explosion (e.g., Mazzali et al. 2005), or possibly of a combination of these two situations.

Available evidence suggests that HVFs are more common before maximum. To obtain early coverage of nearby $\mathrm{SNe}$ Ia is one of the aims of an EU-funded Research Training Network (RTN), linking most European researchers working on SNe Ia. The RTN has collected data for a number of SNe Ia, seven of which have spectra earlier than 1 week before $B$ maximum.

In this Letter, we review all spectra earlier than -7 days to look for evidence of HVFs and assess the ubiquity of this phenomenon. We define an HVF as any absorption that is at a higher velocity than the corresponding photospheric line. A detached HVF is one that is not blended with the photospheric line and is likely to be due to material that is physically detached from the photospheric region. In $\S 2$, we present the 


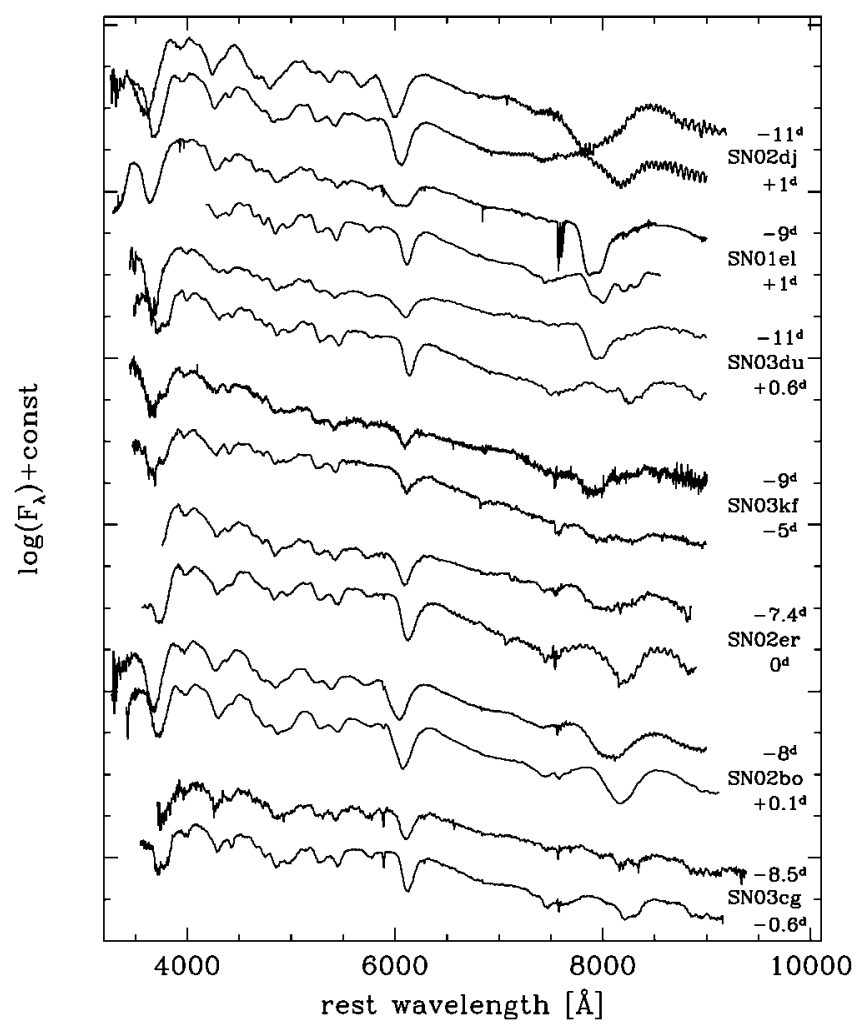

FIG. 1.-Spectra of the SNe Ia observed by the RTN: for each SN, the earliest spectrum is shown, together with a spectrum near maximum. The spectra have been corrected for reddening, arbitrarily scaled in flux, and are ordered by line velocity. Atmospheric absorption was removed from all spectra except those of SN 2001el.

seven RTN SNe with early spectra, and in $\S 3$, we discuss the existing non-RTN sample. Section 4 contains a discussion of the frequency of HVFs and the physical hypotheses we can make about their ubiquity.

\section{HIGH-VELOCITY FEATURES IN THE RTN OBJECTS}

Figure 1 shows spectra of the seven RTN SNe Ia with early observations. Ca II IR HVFs are evident in many SNe, sometimes detached from the photospheric component, and are typically stronger in earlier spectra (e.g., SNe 2002dj and 2003du). Figure 2 shows the $\mathrm{Ca}$ II IR profiles. Table 1 lists the results of fitting the profiles with two separate Gaussians, representing the two components, following Mazzali et al. (2005). The last column of Table 1 is the ratio of the intensities of the two Gaussians. HVFs are not common in the Si II line. In some objects, however, the shape and position of the line suddenly change, indicating the presence and progressive thinning out of an HVF. Below we discuss each SN in turn.

SN 2001el.-Wang et al. (2003) observed strong, detached Ca II IR at $v \sim 22,000-26,000 \mathrm{~km} \mathrm{~s}^{-1}$ between -4 and +2 days, accompanied by significant line polarization. They interpreted this as an indication of the presence of a clump of material at $v \sim 20,000-26,000 \mathrm{~km} \mathrm{~s}^{-1}$. The HVF was also observed in an earlier spectrum (day -9; Mattila et al. 2005). The two strongest lines in the triplet are not blended in the HVF. A similar situation occurred in SN 1999ee (§ 3). The two lines are also partially unblended in the photospheric component in the near-maximum spectrum, as in SNe 2003du and $2003 \mathrm{cg}$. The flat-bottomed Si II profile at day -9 (Mattila et al. 2005) could be an HVF, as in SN 1990N (§ 3).

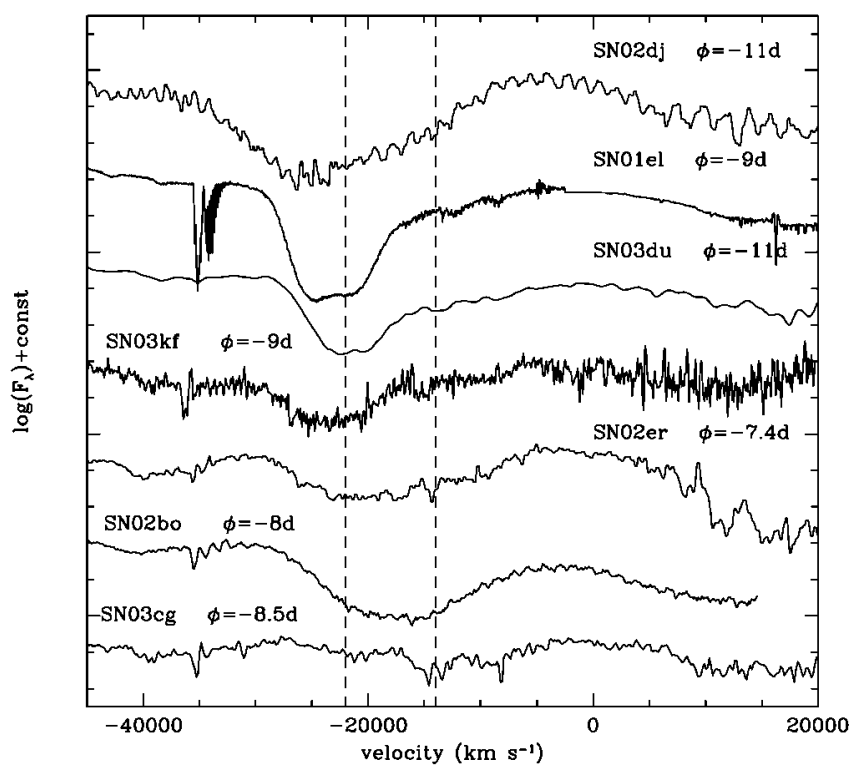

FIG. 2.-Ca II IR triplet profiles in the earliest spectra of the RTN supernovae. The abscissa shows velocity, taking the average wavelength of the multiplet as the zero point, to emphasize the presence of the HVFs. The two vertical lines mark the average position of the photospheric components $\left(14,000 \mathrm{~km} \mathrm{~s}^{-1}\right)$ and of the HVFs $\left(22,000 \mathrm{~km} \mathrm{~s}^{-1}\right)$, respectively.

SN 2002bo.-Benetti et al. (2004) published the spectra of this normal SN Ia. The earliest spectrum covering Ca II IR is at -8.5 days. Although a detached feature is not visible, the line appears blue and broad, becoming narrower and shifting to the red at later epochs. The profile can be decomposed into a high-velocity component near $22,000 \mathrm{~km} \mathrm{~s}^{-1}$ and a photospheric one with similar strength near $15,000 \mathrm{~km} \mathrm{~s}^{-1}$. Stehle et al. (2004) reproduce the profile evolution using a density enhancement at $v \sim 20,000 \mathrm{~km} \mathrm{~s}^{-1}$. As the ejecta expand, the density enhancement becomes optically thin, and by day -3.6 the HVF is very weak. The Si II line profile also changes noticeably, losing strength in the blue between day -11 and maximum, possibly indicative of an HVF.

$S N$ 2002dj.-The earliest red spectrum was observed at -11 days. The $\mathrm{Ca}$ II IR profile is extremely blue, with a central velocity $v \sim 27,600 \mathrm{~km} \mathrm{~s}^{-1}$. The HVF is, however, not detached: the photospheric component, which has a similar

TABLE 1

Properties of the Ca II IR Triplet

\begin{tabular}{|c|c|c|c|c|c|c|}
\hline SN & Day & $\begin{array}{c}v(\mathrm{ph}) \\
\left(\mathrm{km} \mathrm{s}^{-1}\right)\end{array}$ & $\begin{array}{c}W(\mathrm{ph}) \\
\left(\mathrm{km} \mathrm{s}^{-1}\right)\end{array}$ & $\begin{array}{c}v(\mathrm{hv}) \\
\left(\mathrm{km} \mathrm{s}^{-1}\right)\end{array}$ & $\begin{array}{c}W(\mathrm{hv}) \\
\left(\mathrm{km} \mathrm{s}^{-1}\right)\end{array}$ & $F(\mathrm{ph}) / F(\mathrm{hv})$ \\
\hline \multirow[t]{2}{*}{$2002 \mathrm{dj}$} & -11 & 17700 & 11200 & 27600 & 9100 & 1.2 \\
\hline & -3 & 14900 & 11200 & 21800 & 7000 & 17 \\
\hline \multirow[t]{2}{*}{ 2001el ........ } & -9 & 17100 & 14000 & 23800 & 7700 & 0.9 \\
\hline & +1 & 11800 & 8700 & 21400 & 7000 & 0.8 \\
\hline \multirow[t]{2}{*}{$2003 d u$} & -11 & 15500 & 14000 & 22500 & 6600 & 1.3 \\
\hline & +0.6 & 10400 & 8700 & 19300 & 4500 & 4 \\
\hline \multirow[t]{2}{*}{$2003 \mathrm{kf}$} & -9 & 12600 & 14000 & 23500 & 8700 & 0.6 \\
\hline & -5 & 11600 & 9800 & 22100 & 8000 & 0.9 \\
\hline \multirow[t]{2}{*}{ 2002er } & -7 & 15600 & 12200 & 23100 & 6600 & 2.3 \\
\hline & 0 & 13100 & 10500 & 20200 & 6600 & 17 \\
\hline \multirow[t]{2}{*}{2002 bo ....... } & -8 & 14900 & 10500 & 22100 & 8000 & 1.6 \\
\hline & 0.1 & 13100 & 9800 & 18600 & 5600 & 5 \\
\hline \multirow[t]{2}{*}{$2003 \mathrm{cg} \ldots \ldots$} & -8.5 & 12700 & 10100 & 22000 & 4900 & 4.2 \\
\hline & -0.6 & 11700 & 8700 & 19400 & 3500 & 11 \\
\hline
\end{tabular}

Notes. - Velocities are relative to the average Ca II IR triplet wavelength, $8579.1 \AA$, and rounded to the nearest 100. Measurements are more uncertain when the two components are blended (e.g., SN 2002dj). 
strength, is at a high velocity, as might be expected since the epoch of the spectrum is rather early. The photospheric component becomes dominant as the HVF fades: by day -3 , the HVF seems to have disappeared.

SN 2002er.-The earliest Ca II IR spectrum is only at -7.4 days. The line is weaker than in other $\mathrm{SNe}$, but it is very broad. The weakness of both $\mathrm{Ca}$ II IR and $\mathrm{H}$ and $\mathrm{K}$ may indicate a low $\mathrm{Ca}$ abundance or a high ionization degree. Nevertheless, the Ca II IR triplet can be decomposed into an HVF $(v \sim$ $\left.23,000 \mathrm{~km} \mathrm{~s}^{-1}\right)$ and a stronger photospheric component $(v \sim$ $15,000 \mathrm{~km} \mathrm{~s}^{-1}$ ). The HVF has practically disappeared in the spectrum near maximum.

SN 2003cg.-The earliest spectrum covering Ca II IR is at -8.5 days. All $\mathrm{Ca}$ II lines are extremely weak in this $\mathrm{SN}$, deserving some investigation. A weak HVF is visible in the Ca II IR triplet at $v \sim 22,000 \mathrm{~km} \mathrm{~s}^{-1}$. The HVF is detached owing not so much to its velocity but rather to the weakness of the photospheric component (near $12,000 \mathrm{~km} \mathrm{~s}^{-1}$ ), where the two strongest lines of the triplet are unblended. The HVF disappears by the time of maximum.

SN 2003du.-Gerardy et al. (2004) measured an HVF at $v \sim 18,000 \mathrm{~km} \mathrm{~s}^{-1}$ and suggest it is due to the accumulation of circumstellar material (CSM). Our first Ca II IR spectrum is very early, -11.5 days, and it shows a very strong HVF, detached at $v \sim 22,500 \mathrm{~km} \mathrm{~s}^{-1}$. The two strongest lines are not completely blended, indicating some degree of velocity confinement. The photospheric component is weak at first but dominates on day +0.6 , when a weak HVF may still be present: at this epoch, the profile resembles SN 2003cg. The Si II line evolves as in SN 2002bo.

SN 2003kf.-In the earliest spectrum ( -9 days), Ca II IR shows a strong, detached HVF at $v \sim 23,500 \mathrm{~km} \mathrm{~s}^{-1}$ and a weak photospheric component. On day -5 , however, the two components have a similar strength.

In summary, all seven RTN SNe with early spectra show HVFs in Ca II IR; SNe 2001el, 2002bo, and 2003du also show HVFs in the Si II line. Ca II IR HVFs are detached if the photospheric component is weak; otherwise, they broaden the photospheric absorption to the blue. Si II HVFs are much weaker and never detached. HVFs lose strength with time: in most cases, their presence near maximum is doubtful.

\section{EARLY OBSERVATIONS OF OTHER SNe Ia}

Several other SNe Ia have been observed at very early times by different observers in the last 20 years. Here we review the available data and discuss the evidence for HVFs.

SN 1984A.-The very high Si II velocities of this SN, reaching $\sim 16,500 \mathrm{~km} \mathrm{~s}^{-1}$ (Benetti et al. 2005), may be indicative of an HVF. Spectral coverage starts as early as -7 days (Barbon et al. 1989), but unfortunately the Ca II IR region is not covered.

SN 1990N.-The earliest spectrum ( -14 days Leibundgut et al. 1991) does not cover Ca II IR fully. An absorption near $8000 \AA$ may be an HVF. The flat absorption trough of the Si II line, however, has been the subject of debate. Fisher et al. (1997) suggested that it was due to high-velocity C II. Mazzali (2001) showed that high-velocity Si II is a more physical possibility. A similar Si II profile was observed in SNe 2001el and 1999ee (see below).

SN 1991T.-This peculiar object, the prototype of a subclass of SNe Ia, has very early spectra (starting at -12 days) that extend well into the red (Ruiz-Lapuente et al. 1992). However, the ionization conditions must have been so high in the premaximum phase that neither $\mathrm{Ca}$ II nor $\mathrm{Si}$ II lines were visible
(Mazzali et al. 1995). The only strong lines at very early epochs were $\mathrm{Fe}$ III, and these did not show clear signs of HVFs.

SN 1992A.- The red part of the earliest spectrum published by Kirshner et al. (1993) was taken 7 days before maximum. The blue absorption wing of $\mathrm{Ca}$ II IR is not reproduced by the synthetic spectra and may be due to an HVF at $\sim 22,000 \mathrm{~km} \mathrm{~s}^{-1}$. According to Kirshner et al. (1993), Si extends out to at least $19,000 \mathrm{~km} \mathrm{~s}^{-1}$.

SN 1994D.-HVFs were noticed by Hatano et al. (1999) at day -8.9 . Hatano et al. (1999) showed through spectral modeling that $\mathrm{Ca}$ II and $\mathrm{Fe}$ II are present at $25,000 \mathrm{~km} \mathrm{~s}^{-1}<v<$ $40,000 \mathrm{~km} \mathrm{~s}^{-1}$, detached from the photospheric component $\left(v<16,000 \mathrm{~km} \mathrm{~s}^{-1}\right)$. The Ca II IR profile is very similar to that of SN $2003 \mathrm{kf}$ at -9 days. The rapid fading of the HVF in SN $2003 \mathrm{kf}$ suggests that in both SNe HVFs may have been much stronger earlier on.

SN 1998bu.-The earliest spectrum ( -6.8 days; Hernandez et al. 2000) shows a weak Ca II IR detached feature at $v \sim$ $20,000 \mathrm{~km} \mathrm{~s}^{-1}$, which may be weakly present until maximum.

SN 1999aa.-This very slow-declining SN was spectroscopically similar to SN 1991 T at very early times and then rapidly transitioned to normal-looking spectra. The earliest spectrum ( -11 days) showed only very few lines, mostly of Fe III. However, Garavini et al. (2004), on the basis of spectral modeling, suggest the presence of HVFs in both $\mathrm{Ca}$ II and $\mathrm{C}$ II lines at $v>20,000 \mathrm{~km} \mathrm{~s}^{-1}$ in the spectra at $-7,-3$, and -1 days. The time evolution of the HVFs may be consistent with $\mathrm{Ca}$ II forming a clump in the outermost layers of the ejecta.

SN 1999ac.-The premaximum spectra of this SN do not show HVFs. In particular, Ca II IR at -15 days has a minimum at $15,500 \mathrm{~km} \mathrm{~s}^{-1}$ and a blue edge at $30,000 \mathrm{~km} \mathrm{~s}^{-1}$, which may correspond to the photospheric component. However, SN 1999 ac is a peculiar object in many respects. Its spectrophotometric characteristics do not match those of any of the known supernovae (G. Garavini et al. 2005, in preparation).

SN 1999ee.-Mazzali et al. (2005) noticed HVFs in both Ca II IR and Si II $\lambda 6355$, at $v \sim 18,000-20,000 \mathrm{~km} \mathrm{~s}^{-1}$, from -9 to +3 days. The two strongest Ca II IR lines are not blended in the HVF, indicating a narrow velocity confinement of the material, and remain at an almost constant velocity until they disappear. Mazzali et al. (2005) modeled the HVFs using a highvelocity density enhancement, since an abundance enhancement requires that the outer layers are composed only of $\mathrm{Si}$ and $\mathrm{Ca}$. They find that adding $\sim 0.1 M_{\odot}$ of SN composition material can explain the observed HVFs but argue that an outer H-rich layer containing $\sim 0.004 M_{\odot}$ gives an improved solution. HVFs due to $\mathrm{SN}$ composition material would be attributed to a property of the explosion, but the accumulation of $\mathrm{H}$-rich material would necessarily be due to CSM interaction. Both situations may exist in SN 1999ee, the H-rich CSM giving rise to line broadening, and $\mathrm{SN}$ ejecta to the narrow HVFs.

SN 2000cx.-This very peculiar SN shows two very strong sets of detached Ca II IR at different velocities (Li et al. 2001; P. Lundqvist et al. 2005, in preparation). There is no evidence of HVFs in Si II $\lambda 6355$, but Branch et al. (2004) report the presence of Ti II HVFs. Thomas et al. (2004) infer the presence of a detached $\mathrm{Ca}$ II shell at $v>16,000 \mathrm{~km} \mathrm{~s}^{-1}$.

\section{DISCUSSION}

HVFs are present, at least in Ca II IR, in almost all SNe Ia with early spectra, but they have different strength and duration. Some SNe also show Si II HVFs. Spectroscopically, HVFs could be due to abundance or density enhancements. 
An abundance enhancement would be a property of the explosion. However, Mazzali et al. (2005) show that even extreme abundance enhancements, such that $\mathrm{Si}$ and $\mathrm{Ca}$ dominate the outer regions of the ejecta, cannot produce sufficiently strong HVFs. That was a one-dimensional test for SN 1999ee, where the HVFs are not the strongest. Three-dimensional models do show fingers of burned material penetrating to high velocities, but then this material has a covering factor $<1$. Therefore, abundance enhancements alone can only partially explain the observations at best, and density enhancements must also be invoked. Density enhancements would increase the Ca II and Si II line opacity not only directly but also indirectly through recombination in the HVFs ( $\mathrm{Ca}$ and $\mathrm{Si}$ are typically triply ionized in the outermost parts of the ejecta).

Density enhancements may themselves be a property of the explosion: large-scale angular fluctuations, similar to the fingers of burned material in a deflagration, density blobs offering different covering factors depending on the orientation, or the effect of the onset of a delayed detonation. The fact that polarization was observed in coincidence with the HVFs in SN 2001el (Wang et al. 2003) indicates that HVFs are due to threedimensional effects.

Alternatively, density enhancements may be the result of the interaction of the SN ejecta with CSM. In this case, constraints can be placed on the nature of the CSM itself. First, the CSM must offer a large angular size to the ejecta; otherwise, orientation effects would imply that only some SNe should show HVFs. Second, any CSM must be located near the SN for it to affect the spectrum immediately after the explosion. ${ }^{14}$

The CSM closest to the white dwarf (WD) is the accretion disk. If the disk is responsible for the HVFs, it must be thick compared to the size of the WD (Gerardy et al. 2004). Variability in strength and duration of the interaction may depend

\footnotetext{
${ }^{14}$ The strongly interacting SN Ia 2002ic (Hamuy et al. 2003) is an outlier, since interaction starts only after maximum. A different progenitor system may be involved in this case (e.g., an asymptotic giant branch companion).
}

on both disk size and viewing angle. Alternatively, the CSM may be a wind emanating from either star in the system, the strength of the interaction depending on the wind properties. However, for SN 1999ee, Mazzali et al. (2005) find that as much as $0.004 M_{\odot}$ of H-rich material may have accumulated on top of the SN ejecta, and an even larger value was obtained by Gerardy et al. (2004) for SN 2003du. The implied wind mass-loss rate seems unreasonably high for a fast WD wind $\left(\sim 10^{-2} M_{\odot} \mathrm{yr}^{-1}\right)$, but it is high also for a slow red giant wind $\left(\sim 10^{-4} M_{\odot} \mathrm{yr}^{-1}\right)$. Since the material must surround the system, one possibility is enhanced mass loss from the system through the $L_{2}$ or $L_{3}$ points. This should take place at a much higher rate than in single stars.

One property that would distinguish a density enhancement resulting from the explosion from one due to CSM is the composition of the added material. Mazzali et al. (2005) show that the narrow, unblended Ca II IR HVFs in SN 1999ee can be reproduced with a density enhancement of SN composition material but that $\mathrm{H}$-rich material helps to reproduce the overall line width, so this may be a case where the two effects coexist. Unblended HVFs are also observed in SN 2001el. The case of SN 2000cx, with a double set of HVFs, is also interesting. Possibly, the unblended HVFs are due to blobs of material generated in the explosion, while broad HVFs are the result of CSM accretion. If the three-dimensional structure of the ejecta is such that the density profile varies with direction, it may be expected that the range of velocities of the swept-up CSM could be broader than that of the blobs. A study of the composition of the CSM could reveal the nature of SN Ia progenitors.

Early and frequent observations, both spectroscopic and polarimetric, and a study of the correlation between HVF strength and polarization (e.g., SNe 2001el, 2004dt; Wang et al. 2003, 2004, respectively), may reveal what the HVFs imply.

This work was partly supported by the European Research and Training Network 2002-2006 "The Physics of Type Ia Supernovae" (contract HPRN-CT-2002-00303).

\section{REFERENCES}

Barbon, R., Iijima, T., \& Rosino, L. 1989, A\&A, 220, 83

Benetti, S., et al. 2004, MNRAS, 348, 261 2005, ApJ, in press

Branch, D., et al. 2004, ApJ, 606, 413

Fisher, A., Branch, D., Nugent, P. E., \& Baron, E. 1997, ApJ, 481, L89

Garavini, G., et al. 2004, AJ, 128, 387

Gerardy, C. L., et al. 2004, ApJ, 607, 39

Hamuy, M., et al. 2003, Nature, 424, 651

Hatano, K., Branch, D., Fisher, A., Baron, E., \& Filippenko, A. V. 1999, ApJ, 525,881

Hernandez, M., et al. 2000, MNRAS, 319, 223

Kirshner, R. P., et al. 1993, ApJ, 415, 589

Leibundgut, B., et al. 1991, ApJ, 371, L23

Lentz, E. J., Baron, E., Branch, D., Hauschildt, P. H., \& Nugent, P. E. 2000, ApJ, 530, 966

Li, W., et al. 2001, PASP, 113, 1178
Mattila, S., et al. 2005, A\&A, submitted (astro-ph/0501433)

Mazzali, P. A. 2001, MNRAS, 321, 341

Mazzali, P. A., Danziger, I. J., \& Turatto, M. 1995, A\&A, 297, 509

Mazzali, P. A., \& Schmidt, B. P. 2000, in IAU Symp. 201, New Cosmological Data and the Values of the Fundamental Parameters, ed. A. Lasenby, A. Wilkinson, \& A. W. Jones (San Francisco: ASP), 22

Mazzali, P. A., et al. 2005, MNRAS, 357, 200

Riess, A., et al. 1999, AJ, 118, 2675

Ruiz-Lapuente, P., Cappellaro, E., Turatto, E., Gouiffes, C., Danziger, I. J., Della Valle, M., \& Lucy, L. B. 1992, ApJ, 387, L33

Stehle, M., Mazzali, P. A., Benetti, S., \& Hillebrandt, W. 2004, MNRAS, submitted (astro-ph/0409342)

Thomas, R. C., Branch, D., Baron, E., Nomoto, K., Li, W., \& Filippenko, A. V. 2004, ApJ, 601, 1019

Wang, L., et al. 2003, ApJ, 591, 1110 2004, ApJ, submitted (astro-ph/0409593) 